\title{
Evidencia morfológica e isoenzimática de hibridación natural entre Nothofagus antarctica y $N$. pumilio en el noroeste patagónico
}

\author{
Morphological and isozyme evidence of natural hybridization between \\ Nothofagus antarctica and N. pumilio in northwestern Patagonia \\ PAULA QUIROGA ${ }^{1}$, ROMINA VIDAL RUSSELL ${ }^{2}$, ANDREA C. PREMOLI ${ }^{1}$ \\ ${ }^{1}$ Centro Regional Bariloche. Universidad Nacional del Comahue, C.P.8400 Bariloche, Argentina. \\ E-mail: pquiroga@crub.uncoma.edu.ar/apremoli@crub.uncoma.edu.ar \\ 2 Plant Biology Department, Southern Illinois University at Carbondale, Carbondale, Il 62901, U.S.A.
}

\begin{abstract}
SUMMARY
We studied the possible hybridization between $N$. pumilio and N. antarctica by analyzing morphological and isozyme characteristics of a high altitude population at Laguna Negra in northwestern Patagonia, Argentina. Samples collected from this population for morphological and genetic analyses were compared to pure populations of $N$. pumilio and $N$. antarctica. Pure species were statistically different from each other in leaf characteristics. A multivariate analysis indicated greater similarity between the hybrids and $N$. antarctica. Polymorphism at Laguna Negra for 13 loci was 61\% which was higher than that for N. antarctica and N. pumilio (58 and $43 \%$, respectively). We found diagnostic alleles of each pure species and the hybrids resulted similar to $N$. antarctica. These results indicate that natural hybridization between $N$. antarctica and N. pumilio, and introgression into $N$. antarctica take place in Laguna Negra.
\end{abstract}

Key words: hybrids, introgression, Nothofagus antarctica, Nothofagus pumilio, South America.

\section{RESUMEN}

En este trabajo se estudió la hibridación entre Nothofagus pumilio y N. antarctica mediante análisis de morfología foliar y de electroforesis isoenzimática en la población de altura de Laguna Negra en el noroeste de la Patagonia, Argentina. Muestras colectadas en esta población para análisis morfológicos y genéticos fueron comparadas con poblaciones puras de $N$. antarctica y $N$. pumilio. Las especies puras resultaron estadísticamente diferentes entre sí para las variables morfológicas, y el análisis multivariado mostró un patrón de agrupamiento de los híbridos con $N$. antarctica. El polimorfismo para 13 loci en Laguna Negra fue del $61 \%$ y resultó mayor que el de $N$. antarctica y $N$. pumilio (58 y $43 \%$ respectivamente). Se encontraron alelos diagnósticos de las especies puras y los híbridos resultaron más similares a $N$. antarctica. Estos resultados indican que ocurre hibridación natural entre $N$. antarctica y N. pumilio en la población Laguna Negra y la existencia de posibles eventos de introgresión hacia N. antarctica.

Palabras clave: híbridos, introgresión, Nothofagus antarctica, Nothofagus pumilio, Sudamérica.

\section{INTRODUCCION}

La hibridación interespecífica es un fenómeno frecuente en plantas y la facilidad con la que ocurre entre especies arbóreas se debe particularmente a su gran longevidad y a la existencia de varia- dos sistemas reproductivos $(1,2)$. Sin embargo, la formación de híbridos interespecíficos dependerá de la compatibilidad genética entre las dos especies, de la fenología de la floración (3), de la superposición de sus rangos de distribución, y de las condiciones de sitio adecuadas para el estable- 
cimiento de la progenie híbrida (4). Anderson (5) postuló que, para que los híbridos puedan establecerse y sobrevivir, deberían desarrollarse en un "hábitat híbrido". Estos son sitios muchas veces disturbados donde las especies puras tendrían disminuida su adaptabilidad. Además, la reducida competencia entre las especies puras en estos sitios aumentaría las probabilidades de establecimiento de los individuos híbridos. Desde el punto de vista ecológico y evolutivo, la hibridación tiene importancia en cuanto a que contribuye considerablemente a la modificación y al aumento de la diversidad (6) biológica y genética.

Existen numerosos antecedentes de hibridación natural entre especies arbóreas como, por ejemplo, en Pináceas (7), en Quercus (4), y en Nothofagus de Nueva Zelandia (8) y de Sudamérica (9-17). La amplia evidencia de hibridación interespecífica en Nothofagus sugiere que este es un fenómeno extendido en el género a tal punto, que se han descrito especies como Nothofagus leoni (10), cuyo origen se considera híbrido entre N. obliqua y $N$. glauca.

Si bien se ha sugerido que $N$. antarctica (G. Forst.) Oersti y N. pumilio (Poepp. et Endl.) Krasser (Fagaceae) podrían hibridar en condiciones naturales (18), este fenómeno no ha sido descrito hasta el momento. La cercana relación filogenética entre $N$. antarctica y $N$. pumilio (19-21), juntamente con el hecho que coexisten en simpatría en el bosque Andino Patagónico, sugerirían que existe un alto potencial de hibridación entre ellas. En este trabajo mostramos evidencia morfológica e isoenzimática de híbridos entre las especies $N$. pumilio y $N$. antarctica en una población altoandina del noroeste de la Patagonia, Argentina.

\section{MATERIAL Y METODOS}

Se estudió una pequeña población localizada a orillas de la Laguna Negra, ubicada a $1.600 \mathrm{~m}$ s.n.m. sobre el cerro Negro al oeste del Parque

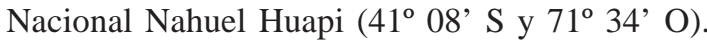
Esta población se ubica en el límite altitudinal de vegetación arbórea y está constituida por $N$. pumilio, N. antarctica y los supuestos híbridos. La altura de todos los individuos no fue superior a los dos metros. En este sitio se detectaron a simple vista posibles individuos híbridos entre
$N$. pumilio y $N$. antarctica a base de la presencia de características morfológicas intermedias entre ambas especies puras. Estas fueron la forma de la hoja, que se diferenció en cordada y elíptico-ovada; el margen de la hoja en aserrado y lobulado; las venas en difusas y marcadas, y los frutos con semillas múltiples o con semilla única. Se tomó un total de 21 muestras, que correspondieron a once individuos híbridos, cuatro individuos de $N$. antarctica y seis individuos de N. pumilio, las cuales se utilizaron para realizar análisis morfológicos y genéticos mediante electroforesis isoenzimática. Los individuos que se identificaron como híbridos fueron comparados con poblaciones puras de $N$. antarctica ubicadas en Lago Steffen $\left(41^{\circ}\right.$ $30^{\prime} \mathrm{S}$ y $71^{\circ} 30^{\prime} \mathrm{O}$ ), Lago Guillelmo ( $41^{\circ} 20^{\prime} \mathrm{S}$ y $71^{\circ} 30^{\prime} \mathrm{O}$ ) y Lago Espejo (403' $\mathrm{S}$ y $71^{\circ} 42^{\prime} \mathrm{O}$ ) estudiadas por Vidal Russell (22) con anterioridad, y puras de $N$. pumilio localizadas en el Valle del Challhuaco ( $41^{\circ} 15^{\prime} \mathrm{S}^{\circ}$ y $\left.71^{\circ} 33^{\prime} \mathrm{O}\right)$ y Cerro López ( $41^{\circ} 15^{\prime}$ S y $71^{\circ} 18^{\prime} \mathrm{O}$ ) estudiadas previamente por Premoli (23), y de los individuos puros de cada especie recolectados en Laguna Negra.

Análisis morfológico. El análisis morfológico se realizó sobre muestras de cinco hojas de disposición no apical por individuo, para 10 individuos de cada una de las tres poblaciones puras de $N$. antarctica, 15 individuos de $N$. pumilio para la población pura de Challhuaco, cuatro para la población pura de Cerro López y los 21 individuos totales de Laguna Negra. Los individuos puros de esta última población se analizaron juntamente con sus respectivos ejemplares de las poblaciones puras. Las variables que se midieron fueron las mismas utilizadas en los trabajos de Romero (24) para Nothofagus sudamericanos y de Ramírez y colaboradores (25) para el análisis de distintos morfotipos de $N$. antarctica. Además, las variables medidas trataron de reflejar la variación cualitativa observada a simple vista de la forma y el margen de la hoja. Las diferencias morfológicas se analizaron comparando los individuos híbridos con individuos puros de Laguna Negra y de las poblaciones mencionadas anteriormente.

Se calculó un promedio por individuo y luego un único valor por especie; posteriormente las variables morfológicas se analizaron mediante análisis de la varianza de una vía y de comparaciones múltiples a posteriori de Tukey (para $\mathrm{N}$ desiguales) corregido por el test de Bonferroni 
BOSQUE 26(2): 25-32, 2005 Evidencia morfológica e isoenzimática de hibridación entre $N$. antarctica y $N$. pumilio en el noroeste patagónico

(26). Sobre éstas se realizó, además, un análisis multivariado de componentes principales (Statistica 5.1 1998).

Análisis isoenzimático. La caracterización genética se realizó mediante la técnica de electroforesis sobre geles de almidón (12\% P/V). Muestras de follaje fresco fueron tomadas de los 21 individuos recolectados en la población híbrida en marzo de 2000. La extracción de enzimas siguió el protocolo descrito en Premoli (14) para Nothofagus. Los homogenatos fueron conservados a $-80^{\circ} \mathrm{C}$ hasta el momento de la corrida electroforética. Se resolvieron 6 sistemas enzimáticos que codificaron para 13 loci génicos putativos. Estos fueron: alcohol deshidrogenasa (Adh-1, Adh-2), aldolasa (Ald-1, Ald-2), isocitrato deshidrogenasa (Idh-1, Idh -2), malato deshidrogenasa (Mdh-1, Mdh-2, Mdh-3, $M d h-4)$, fosfoglucoisomerasa (Pgi-1, Pgi-2), y shikimato deshidrogenasa $(S k d h)$. Todas las enzimas fueron analizadas utilizando el sistema buffer de Morfolina-citrato (MC) a pH 7,5 (27) y los geles fueron corridos a $25 \mathrm{~mA}$ durante 5 horas aproximadamente.

Los datos isoenzimáticos de Laguna Negra fueron comparados con datos de estudios realizados sobre las poblaciones puras de $N$. antarctica (22) y $N$. pumilio (23) indicadas anteriormente. Para realizar el contraste se utilizaron las enzimas polimórficas en común con los estudios de Premoli (24) y Vidal Russell (23) (Adh-1, $A d h-2, I d h-2, M d h-2, M d h-3$ y Pgi-2). Las demás enzimas $I d h-1, M d h-1, M d h-4$ y $P g i-1$ resultaron monomórficas en los tres grupos de poblaciones. A partir de los fenotipos electroforéticos se calcularon frecuencias alélicas, genotípicas y distintos indicadores de variabilidad isoenzimática, utilizando los programas Fstat v. 2.9.3 (28) y Pop Gene 32 v. 1.31 (29). Los índices calculados fueron el número medio de alelos por locus (A), el número de alelos totales $\left(\mathrm{A}_{\mathrm{T}}\right)$, el número promedio de alelos por locus polimórfico $\left(A_{P}\right)$, el porcentaje de loci polimórficos usando el criterio sensu stricto $(\mathrm{P})$, la heterocigosis promedio observada $\left(\mathrm{H}_{\mathrm{O}}\right)$ y la heterocigosis esperada bajo la condición de equilibrio Hardy-Weinberg $\left(\mathrm{H}_{\mathrm{E}}\right)$. La distancia genética entre los individuos puros e híbridos analizados se estimó mediante el índice de Rogers (30).

\section{RESULTADOS}

Análisis morfológico. Los caracteres morfológicos analizados mostraron diferencias significativas tanto entre las especies puras como entre ellas y los supuestos individuos híbridos analizados (cuadro 1). Las especies puras resultaron marcadamente diferentes entre sí para las variables largo total, ancho máximo, largo mínimo y la relación largo total/largo mínimo. Para estos caracteres los híbridos resultaron de morfología foliar similar a $N$. antarctica. La variable relación largo total/largo mínimo puede tomarse como indicador de la forma de la hoja. Así, las hojas de N. pumilio son más redondeadas que las de $N$. antarctica y las de los híbridos que son más acorazonadas. Además, N. antarctica y N. pumilio difirieron significativamente para las variables número de lóbulos marginales entre la segunda y tercera vena lateral y la cantidad de venas laterales. Sin embargo, para estas dos características, los híbridos fueron significativamente distintos a las especies puras, mostrando valores inferiores o intermedios, respectivamente (cuadro 1). Estos resultados muestran que las variables morfológicas largo total, ancho máximo y largo mínimo, como así también el número de lóbulos marginales entre la segunda y tercera vena lateral y la cantidad de venas laterales pueden ser utilizados para identificar y distinguir a $N$. pumilio de $N$. antarctica. Por otro lado, las relaciones largo total/ancho máximo y largo total/número de venas no mostraron diferencias entre los individuos supuestamente híbridos y las especies puras.

El análisis de componentes principales muestra dos grupos bien diferenciados. Uno de estos corresponde a los ejemplares de $N$. pumilio y el otro al agrupamiento de los individuos híbridos con los de $N$. antarctica (fig. 1). Los primeros dos componentes explican el $82,7 \%$ de la varianza total, repartiéndose en $65,3 \%$ y $17,4 \%$ respectivamente. La dispersión de los puntos correspondientes a ambas especies puras posiblemente se deba a diferencias genéticas y/o plasticidad intraespecífica.

Análisis isoenzimático. El análisis isoenzimático para la población de Laguna Negra permitió resolver 13 loci de los cuales 8 resultaron polimórficos (61\% polimorfismo) para al menos uno de los dos grupos de especies puras y supuestos 
BOSQUE 26(2): 25-32, 2005

Evidencia morfológica e isoenzimática de hibridación entre $N$. antarctica y $N$. pumilio en el noroeste patagónico

\section{CUADRO 1}

Variables morfológicas promedio en 11 individuos híbridos y cuatro puros de $N$. antarctica y seis puros de $N$. pumilio analizados junto con 30 y 19 individuos de las especies puras respectivamente. Letras diferentes indican diferencias significativas para el test a posteriori de Tukey con corrección de Bonferroni $P<0,01667$.

Los desvíos estándar se indican en paréntesis.

Morphological characters measured on 11 hybrids and four pure individuals of $N$. antarctica and six pure individuals of $N$. pumilio analyzed together (30 and 19 individuals from pure species respectively). Different letters indicate significant differences, Tukey post-hoc test, $P<0.01667$ after Bonferroni correction. Standard deviations are shown in parenthesis.

\begin{tabular}{|c|c|c|c|c|c|c|c|c|}
\hline & $\begin{array}{l}\text { Largo } \\
\text { total } \\
(\mathrm{mm})\end{array}$ & $\begin{array}{l}\text { Ancho } \\
\text { máximo } \\
(\mathrm{mm})\end{array}$ & $\begin{array}{l}\text { Largo } \\
\text { mínimo } \\
(\mathrm{mm})\end{array}$ & $\begin{array}{c}\mathrm{N}^{\mathrm{o}} \text { de } \\
\text { venas } \\
\text { laterales }\end{array}$ & $\begin{array}{c}\text { Lóbulos } \\
\text { marginales entre } \\
\text { la } 2^{\mathrm{a}} \text { y } 3^{\mathrm{a}} \\
\text { vena lateral }\end{array}$ & $\begin{array}{l}\text { Relación } \\
\text { largo total/ } \\
\text { ancho } \\
\text { máximo }\end{array}$ & $\begin{array}{l}\text { Relación } \\
\text { largo total/ } \\
\text { largo } \\
\text { mínimo }\end{array}$ & $\begin{array}{c}\text { Relación } \\
\text { largo total/ } \\
\mathrm{N}^{\circ} \text { venas } \\
\text { laterales }\end{array}$ \\
\hline $\begin{array}{l}N . \text { antarctica } \\
\mathrm{N}=34\end{array}$ & $\begin{array}{c}15,63^{\mathrm{a}} \\
( \pm 4,67)\end{array}$ & $\begin{array}{c}11,94^{\mathrm{a}} \\
( \pm 2,85)\end{array}$ & $\begin{array}{c}5,57^{\mathrm{a}} \\
( \pm 1,83)\end{array}$ & $\begin{array}{c}4,12^{\mathrm{a}} \\
( \pm 0,67)\end{array}$ & $\begin{array}{c}5,70^{\mathrm{a}} \\
( \pm 1,33)\end{array}$ & $\begin{array}{c}1,33^{\mathrm{a}} \\
( \pm 0,19)\end{array}$ & $\begin{array}{c}3,02^{\mathrm{a}} \\
( \pm 0,51)\end{array}$ & $\begin{array}{c}3,8 \\
( \pm 0,78)\end{array}$ \\
\hline $\begin{array}{l}\text { Híbridos } \\
\mathrm{N}=11\end{array}$ & $\begin{array}{c}13,75^{\mathrm{a}} \\
( \pm 2,50)\end{array}$ & $\begin{array}{c}11,92^{\mathrm{a}} \\
( \pm 2,41)\end{array}$ & $\begin{array}{c}5,35^{\mathrm{a}} \\
( \pm 1,46)\end{array}$ & $\begin{array}{c}3,32^{c} \\
( \pm 0,68)\end{array}$ & $\begin{array}{c}4,45^{\mathrm{c}} \\
( \pm 0,96)\end{array}$ & $\begin{array}{c}1,18^{\mathrm{a}} \\
( \pm 0,23)\end{array}$ & $\begin{array}{c}2,71^{\mathrm{a}} \\
( \pm 0,57)\end{array}$ & $\begin{array}{c}4,40 \\
( \pm 0,47)\end{array}$ \\
\hline $\begin{array}{l}\text { N. pumilio } \\
\mathrm{N}=25\end{array}$ & $\begin{array}{c}20,16^{\mathrm{b}} \\
( \pm 5,73)\end{array}$ & $\begin{array}{c}14,90^{\mathrm{b}} \\
( \pm 4,30)\end{array}$ & $\begin{array}{c}9,72^{\mathrm{b}} \\
( \pm 2,90)\end{array}$ & $\begin{array}{c}5,44^{\mathrm{b}} \\
( \pm 0,58)\end{array}$ & $\begin{array}{c}2^{\mathrm{b}} \\
( \pm 0)\end{array}$ & $\begin{array}{c}1,36^{\mathrm{a}} \\
( \pm 0,12)\end{array}$ & $\begin{array}{c}2,08^{\mathrm{b}} \\
( \pm 0,17)\end{array}$ & $\begin{array}{c}3,70 \\
( \pm 0,87)\end{array}$ \\
\hline$P(\mathrm{ANOVA})$ & 0,0008 & 0,0084 & 0,0000 & 0,0000 & 0,0000 & 0,0321 & 0,0000 & $0,0520 \mathrm{NS}$ \\
\hline
\end{tabular}

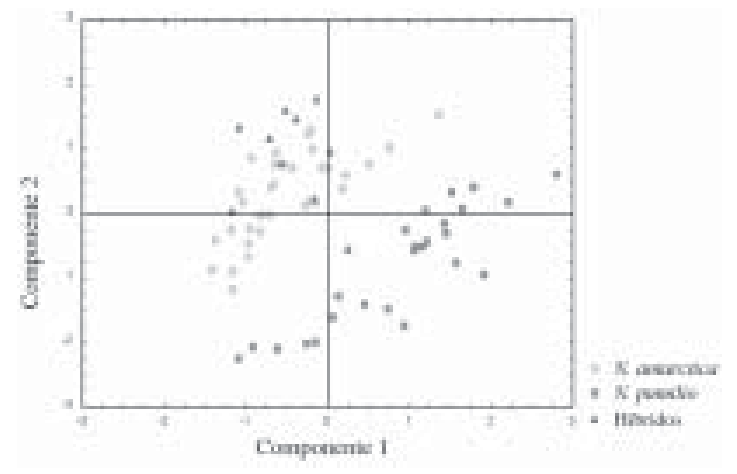

Figura 1. Análisis de Componentes Principales de caracteres foliares para individuos híbridos y puros de $N$. antarctica y N. pumilio de Laguna Negra y de las otras poblaciones puras analizadas (la ubicación de las mismas puede encontrarse en el texto).

Principal Component Analysis of leaf characteristics for hybrid individuals and pure $N$. antarctica and $N$. pumilio from Laguna Negra and the other analyzed pure populations (for location of these populations see text).

híbridos. Los loci polimórficos resueltos para Laguna Negra fueron Adh-1, Adh-2, Ald-1, Idh-2, $M d h-2, M d h-3, P g i-2$ y $S k d h$. Este grado de polimorfismo resultó mayor que el encontrado en las poblaciones puras de $N$. pumilio y $N$. antarctica estudiadas con anterioridad, cuyos valores fueron del $43 \%$ sobre un total de 14 loci (23) y $58 \%$ sobre un total de 12 loci (22).

Las especies puras pueden distinguirse entre sí por la presencia de alelos diagnósticos para 4 loci analizados (Adh-1,1; Mdh-2,4; Mdh-3,1 y Pgi2,1) (cuadro 2). Además, en la población de Laguna Negra se observa que existen alelos compartidos entre los híbridos y una de las especies puras, como es el caso de $A d h-1,3$ y $A d h-2,3$ con $N$. antarctica y $M d h-2,1$ y $M d h-3,3$ con N. pumilio (cuadro 2). Sin embargo, dos alelos, $M d h-2,2$ y $M d h-2,3$, resultaron con marcadas diferencias en sus frecuencias para las especies puras (fig. 2). Los individuos híbridos presentaron mayor heterocigosis que las especies puras, a pesar de haber analizado un reducido número de éstos (cuadro 3). Las especies puras resultaron similares genéticamente entre sí con una distancia genética de Rogers de 0,905. Por otro lado, las distancias genéticas de los híbridos con $N$. antarctica y $N$. pumilio resultaron de 0,902 y 0,831 respectivamente. La menor distancia genética calculada entre los híbridos y $N$. antarctica refleja frecuencias alélicas similares entre estos grupos de individuos (cuadro 2). 
BOSQUE 26(2): 25-32, 2005 Evidencia morfológica e isoenzimática de hibridación entre $N$. antarctica y $N$. pumilio en el noroeste patagónico

\section{CUADRO 2}

Frecuencias alélicas en seis loci polimórficos para los 11 individuos híbridos de Laguna Negra (LN) y poblaciones puras de $N$. antarctica y $N$. pumilio, analizadas junto con los individuos puros respectivos de Laguna Negra. Los alelos compartidos entre los híbridos con una de las especies puras se indican con asterisco $(*)$ y los alelos diagnósticos de cada especie pura se designan con D.

Alleles frequencies at six polymorphic loci for 11 hybrid individuals from Laguna Negra (LN) and pure populations

of $N$. antarctica and $N$. pumilio, analyzed with pure individuals from Laguna Negra. Shared alleles by hybrids with one pure species are indicated by $(*)$ and those diagnostic alleles of each pure species are designated by D.

\begin{tabular}{|c|c|c|c|}
\hline Loci/alelos & N. antarctica & Híbridos LN & N. pumilio \\
\hline \multicolumn{4}{|l|}{$A d h-1$} \\
\hline $1 \mathrm{D}$ & & & 0,02 \\
\hline 2 & 0,96 & 0,70 & 0,98 \\
\hline $3^{*}$ & 0,04 & 0,30 & \\
\hline \multicolumn{4}{|l|}{$A d h-2$} \\
\hline 1 & 0,01 & & 0,01 \\
\hline 2 & 0,98 & 0,73 & 0,99 \\
\hline $3 *$ & 0,01 & 0,27 & \\
\hline \multicolumn{4}{|l|}{$I d h-2$} \\
\hline 1 & 0,01 & 0,14 & 0,02 \\
\hline 2 & 0,96 & 0,73 & 0,97 \\
\hline 3 & 0,03 & 0,14 & 0,01 \\
\hline \multicolumn{4}{|l|}{$M d h-2$} \\
\hline $1 *$ & & 0,14 & 0,01 \\
\hline 2 & 0,13 & 0,09 & 0,96 \\
\hline 3 & 0,86 & 0,77 & 0,03 \\
\hline $4 \mathrm{D}$ & 0,01 & & \\
\hline \multicolumn{4}{|l|}{$M d h-3$} \\
\hline $1 \mathrm{D}$ & & & 0,03 \\
\hline 2 & 1,00 & 0,91 & 0,96 \\
\hline $3^{*}$ & & 0,09 & 0,02 \\
\hline \multicolumn{4}{|l|}{$P g i-2$} \\
\hline $1 \mathrm{D}$ & & & 0,03 \\
\hline 2 & 0,96 & 1,00 & 0,94 \\
\hline 3 & 0,04 & & 0,03 \\
\hline
\end{tabular}

\section{DISCUSION Y CONCLUSIONES}

La evidencia morfológica e isoenzimática presentada en este trabajo sugiere la existencia de hibridación natural entre $N$. antarctica y $N$. pumi-

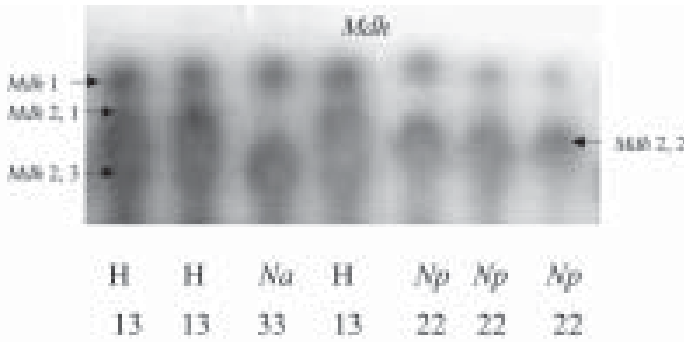

Figura 2. Zimograma de la enzima $M d h$ para individuos híbridos $(\mathrm{H})$ y puros de $N$. antarctica $(\mathrm{Na})$ y $N$. pumilio $(\mathrm{Np})$ de Laguna Negra. Sobre los márgenes se indican las distintas isoenzimas con sus alelos y al pie se muestran los genotipos correspondientes a Mdh-2.

Zimogram of the enzyme $M d h$ for hybrid $(\mathrm{H})$ and pure individuals of $N$. antarctica $(\mathrm{Na})$ and $N$. pumilio $(\mathrm{Np})$ from Laguna Negra. Different isozymes with their alleles are indicated on the sides and genotypes for different individuals for Mdh-2 are shown at the bottom.

lio para una población de altura del noroeste patagónico. Los datos de morfología foliar indican que para aquellos caracteres diagnósticos de las especies puras: los híbridos o poseen valores intermedios (para la variable lóbulos marginales entre la $2^{\mathrm{a}}$ y $3^{\mathrm{a}}$ vena lateral) o no difieren estadísticamente de $N$. antarctica (para las variables largo total, ancho máximo, largo mínimo y para la relación largo total/largo mínimo). La detección de híbridos usando caracteres morfológicos asume que los individuos híbridos podrían ser fenotípicamente intermedios entre los individuos parentales especialmente bajo regímenes de selección estabilizadora (31). Sin embargo, a menudo esto no ocurre, porque los híbridos pueden expresar un mosaico de los fenotipos parentales (32). Los individuos híbridos detectados por Gallo y colaboradores (16) muestran claramente que éstos tienen características morfológicas diferentes a los parentales y que no necesariamente presentan fenotipos intermedios entre los tipos parentales. El grado en que la progenie híbrida difiere de los progenitores dependerá del grado de recombinación y la intensidad de selección (31) Así, en casos donde exista intensa selección direccional se espera que los individuos híbridos presenten cambios tanto en sus características genéticas como fenotípicas. El estadísticamente menor número de venas laterales que presentan los híbridos, juntamente con el agrupamiento de los híbridos dentro de la clase de $N$. antarctica en el análisis de com- 
BOSQUE 26(2): 25-32, 2005

Evidencia morfológica e isoenzimática de hibridación entre $N$. antarctica y $N$. pumilio en el noroeste patagónico

\section{CUADRO 3}

Variabilidad genética para 13 loci en todas las poblaciones analizadas. $\mathrm{N}$ es el número promedio de individuos analizados por locus; A es el número promedio de alelos observado por locus, $\mathrm{A}_{\mathrm{T}}$ es el número de alelos totales; $\mathrm{A}_{\mathrm{P}}$ es el número promedio de alelos por locus polimórfico; P representa el porcentaje de loci polimórficos utilizando el criterio sensu stricto; $\mathrm{H}_{0}$ es la heterocigosis observada, y $\mathrm{H}_{\mathrm{E}}$ es la heterocigosis esperada bajo la condición de equilibrio Hardy-Weinberg. Los errores estándar se muestran entre paréntesis.

Average genetic variability parameters at 13 loci in all analyzed populations. $\mathrm{N}$ is the average number of analyzed individuals per locus, $\mathrm{A}$ is the mean number of alleles per locus, $\mathrm{A}_{\mathrm{T}}$ is the total number of alleles, $\mathrm{A}_{\mathrm{P}}$ is the mean number of alleles per polymorphic locus, $\mathrm{P}$ is the percentage of polymorphic loci using sensu stricto criteria, $\mathrm{H}_{0}$ and $\mathrm{H}_{\mathrm{E}}$ are the observed and expected heterozygosity, respectively. Standard errors are shown in parenthesis.

\begin{tabular}{|c|c|c|c|c|c|c|c|}
\hline & $\mathrm{N}$ & A & $\mathrm{A}_{\mathrm{T}}$ & $\mathrm{A}_{\mathrm{P}}$ & $\mathrm{P}$ & $\mathrm{H}_{0}$ & $\mathrm{H}_{\mathrm{E}}$ \\
\hline $\begin{array}{l}N . \text { antarctica } * \\
\mathrm{~N}=222\end{array}$ & 151 & 1,8 & 18 & 2,6 & 50 & $\begin{array}{l}0,010 \\
(0,004)\end{array}$ & $\begin{array}{l}0,052 \\
(0,024)\end{array}$ \\
\hline $\begin{array}{l}\text { Híbridos LN } \\
\mathrm{N}=11\end{array}$ & 10,9 & 1,7 & 17 & 2,4 & 50 & $\begin{array}{l}0,136 \\
(0,062)\end{array}$ & $\begin{array}{l}0,188 \\
(0,067)\end{array}$ \\
\hline $\begin{array}{l}\text { N. pumilio* } \\
\mathrm{N}=250\end{array}$ & 208,9 & 2,0 & 20 & 2,7 & 60 & $\begin{array}{l}0,022 \\
(0,011)\end{array}$ & $\begin{array}{l}0,042 \\
(0,014)\end{array}$ \\
\hline
\end{tabular}

* Incluye los individuos puros de Laguna Negra.

* Include the pure individuals from Laguna Negra.

ponentes principales reflejaría la existencia de un grupo claramente distinguible. El mismo sería producto de la selección y la hibridación cuya evidencia es reforzada por los resultados isoenzimáticos.

La dispersión de los puntos obtenidos en el análisis de componentes principales de datos morfológicos reflejaría la variación intraespecífica asociada a la amplia distribución latitudinal y altitudinal de $N$. antarctica y $N$. pumilio (fig. 1). N. antarctica posee una variación intraespecífica elevada en respuesta a extremos y variados ambientes que modifican su cuerpo vegetativo, adoptando diferentes formas de vida que varían desde arbóreas y arbustivas a subarbustivas $(25,33)$. Por otro lado, N. pumilio posee una marcada variación intraespecífica a lo largo de gradientes altitudinales en caracteres morfológicos, ecofisiológicos e isoenzimáticos $(23,34)$.

Las especies puras pueden distinguirse isoen- zimáticamente entre sí por la presencia de alelos diagnósticos. Si bien los híbridos resultaron similares a $N$. antarctica, mostraron una mayor heterocigosis. Esto evidencia la presencia de alelos con frecuencias intermedias respecto de las especies puras. Similares resultados isoenzimáticos se obtuvieron al comparar los Nothofagus siempreverdes sudamericanos, donde las distintas especies puras presentaron diferencias en las frecuencias alélicas para varios sistemas isoenzimáticos (14). En otros trabajos también pudieron distinguirse especies puras entre sí y diferenciarse de sus híbridos, basándose en la presencia (o ausencia) de alelos diagnósticos y/o diferencias en las frecuencias alélicas (35-38) incluidas otras especies de Nothofagus sudamericanos (16).

Los caracteres diagnósticos de especies puras pueden aparecer combinados en los híbridos y ser reconocidos durante las primeras generaciones híbridas (5). Luego de esta primera generación, si 
BOSQUE 26(2): 25-32, 2005 Evidencia morfológica e isoenzimática de hibridación entre $N$. antarctica y $N$. pumilio en el noroeste patagónico

bien el promedio de estos caracteres se mantiene constante en la población, se espera que los individuos híbridos sean extremadamente variables en su aspecto morfológico $(5,12)$. Esto fue evidente en algunos de los individuos híbridos de la población Laguna Negra, dado que no se podía distinguir con facilidad la afinidad morfológica con una u otra especie pura. Por lo tanto, estos individuos híbridos no representarían una primera generación híbrida, debido a que muchas veces los caracteres morfológicos no sirven para determinar si un individuo corresponde a una primera generación híbrida, a una retrocruza reciente o a un largo proceso de hibridación (33).

Los individuos híbridos resultaron más similares a $N$. antarctica que a $N$. pumilio. Si bien este fenómeno se detectó sobre un número reducido de individuos híbridos, el mismo podría ser el resultado de sucesivos cruzamientos entre una generación híbrida y el tipo parental $N$. antarctica. Así, a lo largo de las generaciones se incrementan las similitudes respecto de una de las especies parentales, fenómeno que recibe el nombre de introgresión (39). La mayor similitud observada a partir de los datos morfológicos y genéticos sugieren la ocurrencia de introgresión hacia $N$. antarctica. Este fenómeno ha sido también descrito para otras especies de Nothofagus como, por ejemplo, de $N$. obliqua hacia $N$. glauca (10); $N$. alpina hacia $N$. obliqua $(13,16)$, y de $N$. nitida hacia $N$. betuloides $(12,14,15)$. Sin embargo, el número de individuos híbridos y el número de plantas puras analizadas en este trabajo es relativamente bajo. Por lo tanto, se requieren más estudios que analicen la ocurrencia de este fenómeno especialmente en otras áreas donde las dos especies coexisten en simpatría.

Los eventos de hibridación natural entre $N$. antarctica y $N$. pumilio podrían ser más frecuentes que lo considerado hasta el presente. La evidencia presentada en este trabajo juntamente con evidencias del Glaciar Martial en Tierra del Fuego (34) y observaciones realizadas por P. Quiroga y A. Premoli en otros fondos de valles, como el Valle de los Perdidos cercano a Laguna Negra y hacia el sur del bosque templado en el Chaltén, provincia de Santa Cruz y Paso Garibaldi en Tierra del Fuego, sugieren que la hibridación podría estar asociada a condiciones ambientales favorables para la viabilidad de los híbridos. En particular la hibridación entre $N$. antarctica y N. pumilio estaría facilitada por su cercanía filogenética (19-20). Por lo tanto, cuando se solapan los períodos de floración de ambas especies puras y en ausencia de barreras postcigóticas, no existirían restricciones para el flujo génico interespecífico. Esto es particularmente relevante en el gradiente altitudinal, donde se pudo demostrar que N. pumilio presenta marcadas restricciones al flujo génico intraespecífico (34). El aislamiento reproductivo entre individuos separados altitudinalmente por escasos cientos de metros propiciaría el intercambio genético interespecífico con individuos simpátricos de $N$. antarctica. Sin embargo, para facilitar el establecimiento de los híbridos es importante que el ambiente en el cual se desarrollan sea también híbrido, o bien, presente características propias $(5,40)$. En particular, la población de Laguna Negra se encuentra sobre los límites altitudinales de la vegetación arbórea, donde los factores ambientales que la afectan son probablemente diferentes a los que operan en los ambientes donde ocurren las especies puras. El uso de un nuevo ambiente en el gradiente altitudinal, por parte de este grupo de individuos híbridos, puede llevar a la explotación de nuevos recursos que, en combinación con una elevada heterocigosis, permitiría la diferenciación por selección direccional y potencial especiación de las especies caducifolias dentro del subgénero $\mathrm{No}$ thofagus del bosque templado austral.

\section{BIBLIOGRAFIA}

(1) GRANT, V. The evolutionary process. $2^{\text {nd }}$ ed, Columbia University Press. New York, 1991. Isolating mechanisms, p. 206-214.

(2) DONOSO, C. Bosques templados de Chile y Argentina, variación, estructura y dinámica. $3^{\text {ra }}$ ed., Editorial Universitaria, Santiago de Chile, Chile. 1995. Variación de especies forestales y genecología, p. 25-44.

(3) STERN, K., L. ROCHE. Genetics of forest ecosystems. Ecological studies 6. Springer-Verlag, New York. 1974.

(4) MÜLLER, C. Ecological control of hybridization in Quercus: a factor in the mechanism of evolution. Evolution, 1952, vol. 6, p 147-161.

(5) ANDERSON, E. Hybridization of the habitat. Evolution, 1948, vol. 2, p. 1-9.

(6) STEBBINS, G.L. Variation and Evolution in plants. New York, Columbia University Press. 1950.

(7) MIROV, N.T. The genus Pinus. The Royal Press Co. New York. 1967.

(8) POOLE, A.L. The flowering, seeding and hybridization of beeches. New Zealand Forest Service. Beech Forestry in the New Zealand 1964, Symposium 2 (5).

(9) DONOSO, C., L. LANDRUM. Nothofagus leoni hibridación e introgresión en poblaciones de $N$. obliqua y 
BOSQUE 26(2): 25-32, 2005

Evidencia morfológica e isoenzimática de hibridación entre $N$. antarctica y $N$. pumilio en el noroeste patagónico

N. glauca. Boletín Técnico No 36 , Facultad de Ciencias Forestales, Universidad de Chile, Santiago. 1976, p. 4-29.

(10) DONOSO, C., L. LANDRUM. Nothofagus leoni Espinosa a natural hybrid between Nothofagus obliqua (Mirb.) Oerst. and Nothofagus glauca (Phil.) Krasser. New Zealand Journal of Botany, 1979, $\mathrm{N}^{\circ} 17$, p. 353-360.

(11) DONOSO, C., J. ATIENZA H. Hibridación natural entre especies de Nothofagus siempreverdes en Chile. Bosque 1983, vol. 5, $\mathrm{N}^{\circ} 1$, p. 21-34.

(12) DONOSO, C., J. ATIENZA H. 1984. Hibridación natural entre Nothofagus betuloides (Mirb.) Oerst. y Nothofagus nitida (Phil.) Krasser. Medio Ambiente, vol. 7, $\mathrm{N}^{\mathrm{o}} 1$, p. 9-16.

(13) DONOSO. C., J. MORALES, M. ROMERO. Hibridación natural entre roble (Nothofagus obliqua) (Mirb) Oerst. y raulí (Nothofagus alpina) (Poepp. \& Endl.) Oerst, en bosques del sur de Chile. Revista Chilena de Historia Natural, 1990, $\mathrm{N}^{\circ}$ 63, p. 49-60.

(14) PREMOLI, A. Allozyme polymorphisms, outcrossing rates, and hybridization of South American Nothofagus. Genetica, 1996a, vol. 97, p. 55-64.

(15) PREMOLI, A.C. Leaf architecture of South American Nothofagus (Nothofagaceae) using traditional and new methods in morphometrics. Botanical Journal of the Linnean Society, 1996, vol. 121, p. 25-40.

(16) GALLO, L.A., P. MARCHELI, A. BREITEMBÜCHER Morphological and allozymic evidence of natural hybridization between two southern beeches (Nothofagus spp.) and its relation to heterozygosity and height growth Forest Genetics, 1997, vol. 4, No 1 , p. 15-23.

(17) STECCONI, M., P. MARCHELLI, J. PUNTIERI, L. GALLO. Hybridization between Nothofagus antarctica (deciduous) and $N$. dombeyi (evergreen) (Nothofagaceae) in natural communities. Southern Connection Bulletin, 2002, N $\mathrm{N}^{\circ} 21$, p. 8-9.

(18) DONOSO, C. Variación natural en especies de Nothofagus en Chile. Bosque, 1987, vol. 8, No 2, p. 85-97.

(19) HILL, R.S., G.J. JORDAN. The evolutionary history of Nothofagus (Nothofagaceae). Australian Systematic Botany, 1993, vol. 6, p. 111-126.

(20) MARTIN, P.G., J.M. DOWD. Using sequences of rbcL to study phylogeny and biogeography of Nothofagus species. Australian Systematic Botany, 1993, vol. 6 , p. 441-447.

(21) MANOS, P.S. Systematics of Nothofagus (Nothofagaceae) based on rDNA spacer sequences (ITS): Taxonomic congruence with morphology and plastid sequences. American Journal of Botany, 1997, vol. 84, $\mathrm{N}^{\circ} 8$, p. 1137-1155.

(22) VIDAL RUSSELL, R. Evidencias de resistencia en $\mathrm{No}$ thofagus a Misodendrum: patrones de infección y consecuencias sobre la estructura genética de la planta parásita. Tesis de licenciatura. Facultad de Biología. Universidad Nacional del Comahue, Bariloche, Argentina, 2000.

(23) PREMOLI, A.C. Isozyme polymorphisms provide evidence of clinal variation with elevation in Nothofagus pumilio. Journal of Heredity, 2003, vol. 94, No 3 , p. 218-226.

(24) ROMERO, E.J. Arquitectura foliar de las especies sudamericanas de Nothofagus B1. Boletín de la Sociedad Argentina de Botánica, 1980. Vol. XIX, No 1-2, p. 298-308.
(25) RAMÍREZ, C., M. CORREA, H. FIGUEROA, J. SAN MARTÍN. Variación del hábito y hábitat de Nothofagus antarctica en el centro sur de Chile. Bosque, 1985, vol. 6, $\mathrm{N}^{\mathrm{0}}$ 2, p. 55-73.

(26) RICE, W.R. Analyzing tables of statistical test. Evolution, 1989, vol. 43, No 1, p. 223-225

(27) RANKER, T.A., C.H. HAUFLER, P.S. SOLTIS, D.E. SOLTIS. Genetic evidence for allopolyploidy in the neotropical fern Hemionitis (Adiantaceae) and the reconstruction of an ancestral genome. Systematic Botany, 1989, vol. 14, p. 439-447.

(28) GOUDET, J. FSTAT. A program to estimate and test gene diversities and fixation indices, release 2.9.1. Université de Lausanne, Dorigny, Switzerland, 2002.

(29) YEH, F., R.C. YANG, T. BOYLE. POPGENE $32 \mathrm{v}$ 1.31Microsoft Window-based freeware for population genetic analysis. University of Alberta. Canada, 1999.

(30) ROGERS, J.S. Measures of genetic similarity and phylogenetic distance. Studies in genetics. University of Texas, 1972, Publ. 7213, p. 145-153.

(31) SOLBRIG, Q. T.,D. J. SOLBRIG. Introduction to populations biology and evolution. Addison - Wesley Pub. Co. 1979. Chapter 12, Hybridization, preadaptation, and adaptation, p. 297-315.

(32) ALLENDORF, F. W., R. F. LEARY, P. SPRUELL, J. K. WENBURG. The problems with hybrids: setting conservation guidelines. TREE, 2001. vol 16, $\mathrm{N}^{\circ} 11,613-$ 622.

(33) PREMOLI, A.C. Morfología y capacidad germinativa en poblaciones de Nothofagus antarctica (Forster) Oerst. del noroeste andino patagónico. Bosque, 1991, vol. 12, $\mathrm{N}^{\mathrm{o}}$ 2, p. 53-59.

(34) PREMOLI, A.C. Variación en Nothofagus pumilio (Poepp. et Endl.) Krasser. En: Donoso, Z.C., A.C. Premoli, L. Gallo, R. Ipinza. Variación en las especies arbóreas de los bosques templados de Chile y Argentina. Editorial Universitaria, Santiago de Chile, 2004, p. 145172.

(35) WHEELER, N.C., R. GURIES. A quantitative measure of introgression between lodgepole and jack pines. $\mathrm{Ca}$ nadian Journal of Botany, 1987, vol. 65, № 9, p. 18761885 .

(36) DePANPHILIS, C.W., R. WYATT. Electrophoretic confirmation of interespecific hybridization in Aesculus (Hippocastanaceae) and the genetic structure of a broad hybrid zone. Evolution, 1990, vol. 44, p. 1295-1317.

(37) MAKI, M., J. MURATA. Allozyme analysis of the hybrid origin of Arisaema ehimense (Araceae). Heredity, 2001, vol. 86, N $\mathrm{N}^{\mathrm{0}} 1$, p. 87-93.

(38) XIAO-RU, W., A.E. SZMIDT, O. SAVOLAINEN. Genetic composition and diploid hybrid speciation of high mountain pine, Pinus densata, native to the Tibetan Plateau. Genetics, 2001, vol. 159, No 1, p. 337-346.

(39) ANDERSON, E., L. HUBRICHT. The evidence for introgressive hybridization. American Journal of Botany, 1938, vol. 25, p. 396-402.

(40) KONSTANTIN, V.K., F. BERGMANN. Introgressive hybridization and phylogenetic relationships between Norway, Picea abies (L.) Karst., and Siberian, P. obovata Ledeb., spruce species studied by isozyme loci. Heredity, 1995, vol. 74, p. 464-480. 